Rev. salud pública. 14 (1): 169-181, 2012

\title{
Prevalencia y factores de riesgo de Cryptosporidium spp. y Giardia spp. en terneros de ganado lechero de la zona noroccidental de la Sabana de Bogotá
}

\section{Cryptosporidium spp. and Giardia spp. prevalence and risk factors in dairy calves of the north-western zone of the Bogota Savanna}

\author{
Nicolás Hernández-Gallo y Jesús A. Cortés-Vecino²
}

1 Consultor Convenio 485/10 Organización Panamericana de la Salud-Ministerio de Salud y de Protección Social.nhernandezg@unal.edu.co

2 Profesor Asociado. Laboratorio de Parasitología Veterinaria. Facultad de Medicina Veterinaria y de Zootecnia, Universidad Nacional de Colombia. jacortesv@unal.edu.co

Recibido 22 Noviembre 2010/Enviado para Modificación 20 Agosto 2011/ Aceptado 15 Noviembre 2011

\section{RESUMEN}

Objetivo Estimar la prevalencia de Cryptosporidium spp. y Giardia spp. en terneros de 0 a 2 meses de ganado lechero de la zona noroccidental de la Sabana de Bogotá. Se estimaron los factores de riesgo de la producción lechera que puedan incurrir en la infección de personas y animales.

Métodos Estudio de corte transversal, mediante la toma de materia fecal de terneros. Se determinó la prevalencia de punto para Giardia spp. y Cryptosporidium spp. a través los resultados de los coprológicos (Ritchie para Giardia spp. y ZiehlNeelsen modificada para Cryptosporidium spp.). Se calcularon Odds Ratio para establecer los factores de riesgo asociados entre estos dos géneros de protozoarios y el manejo de las Buenas Prácticas Ganaderas.

Resultados Se evaluaron 33 fincas dedicadas a la producción lechera, donde se muestrearon 308 terneros. La prevalencia para Giardia spp. fue de 37,3\%, 115 animales positivos y para Cryptosporidium spp. fue de 4,9\% 15, animales positivos.

Conclusión Existe un foco de Giardia spp. y Cryptosporidium spp. en el noroccidente de la Sabana de Bogotá, del cual no se tenía conocimiento previo. La prevalencia de Giardia spp. para la región estudiada, está en el límite alto del rango reportado para Sudamérica. En el caso de Cryptosporidium spp. la situación es distinta, la prevalencia se encuentra en el limite bajo del rango reportado para Sudamérica. Los factores de riesgo asociados a Giardia spp. y Cryptosporidium spp. en las explotaciones lecheras del noroccidente de la Sabana de Bogotá, dependen de Buenas Prácticas Ganaderas. 
Palabras Clave: Zoonosis, prevalencia, factor de riesgo y factor de protección (fuente: DeCS, BIREME).

\section{ABSTRACT}

Objective The present study was aimed to establishing Cryptosporidium spp. and Giardia spp. prevalence in 0 to 2 months old dairy calves of the north-western zone of the Bogota Savanna. In addition, associated factors related to a failure in Good Practices of Livestock could incur in human and animal infection.

Methods This was a cross-sectional study; calves' fecal samples were used. Farms' Good Practices of Livestock were observed by means of an observation blank. Giardia spp. and Cryptosporidium spp. prevalence was determinate by means of laboratory results (Ritchie for Giardia spp. and modified Ziehl-Neelsen for Cryptosporidium spp.). Odds Ratios (OR) were calculated in association between this two genera of protozoa and Good Practices of Livestock.

Results Thirty three dairy farms were evaluated, where fecal samples of 308 calves were taken. Giardia spp. prevalence was $37.7 \%, 115$ infected animals; Cryptosporidium spp. prevalence was $4.9 \%, 15$ infected animals.

Conclusion There is an important Giardia spp. and Cryptosporidium foci in the north-western zone of the Bogota Savanna, without a previous knowledge. Giardia spp. prevalence for this zone is in the highest rank reported for South-America and Cryptosporidium spp. prevalence is in en the lowest one. Associated risk factors of Giardia spp. and Cryptosporidium spp. in dairy farms of the north-western zone of the Bogota Savanna depend of a Good Practices of Livestock performance.

Key Words: Zoonosis, prevalence, risk and protection factors (source: $M e S H$, $N L M)$.

$\mathrm{E}$ $175 \%$ de las enfermedades infecciosas son de naturaleza zoonótica, se sabe que Cryptosporidium y Giardia son parásitos que infectan a todos los mamíferos y han sido problema importante en Salud Pública causando epidemias de diarrea, tanto en humanos como en animales (1). Cryptosporidium parvum es el parásito que ha causado significativas infecciones en humanos desde los últimos diez años del siglo pasado y en los primeros diez de este, especialmente en personas inmunosuprimidas; aunque desde comienzos del siglo XX se conoció su naturaleza zoonótica sólo hasta finales de este empezaron a preocuparse por hacer estudios en animales de compañía y sólo en los dos últimos años del siglo se empezaron a ver reportes en animales de producción (2). Giardia spp es una entidad de la cual se han tenido reportes desde el comienzo del siglo pasado (1914), se han establecido factores de riesgo para humanos y las mascotas, especialmente ha sido una enfermedad asociada a personas de 
bajos recursos y a los perros (3). Hasta comienzos de este siglo (2001) se vuelven a ver estudios y reportes sobre estos dos protozoarios en las fincas dedicadas a la producción pecuaria (4).

La transmisión zoonótica de Cryptosporidium spp. y Giardia spp. de animales domésticos al humano ha sido inferida en algunos reportes por entrar en contacto con las heces de los animales infectados (5).La fuente más común de transmisión zoonótica por estos parásitos está relacionada con el contacto con materia fecal, por la cual se eliminan los quistes de estos parásitos, con el agua destinada a consumo humano o animal. También se relaciona el contacto con heces infectadas de la leche y/o algún otro producto pecuario destinado a consumo humano, donde no se hayan tenido los cuidados sanitarios pertinentes en la producción. Esto es muy común en los países en vía de desarrollo donde la producción pecuaria es todavía artesanal y un bajo porcentaje es tecnificada (6).

Debido a que no se cuenta con suficiente información sobre la prevalencia de Cryptosporidium spp, Giardia spp en el gado lechero perteneciente a la Sabana de Bogotá; el estudio es importante porque es pertinente saber la prevalencia actual en la Sabana de Bogotá de los dos parásitos, en los últimos 20 años han tenido un alto impacto en Salud Pública (2) y la Sabana es una de las regiones de producción lechera más importante en el país (7), es necesario el conocimiento arrojado por este estudio para conocer la prevalencia de los dos parásitos en esta región del país, sus posibles factores de riesgo, establecer fallas en el manejo de las fincas y así contribuir a la preservación de la salud humana.

Las Buenas Prácticas Ganaderas (BPG) se entienden como la aplicación del conocimiento disponible para la utilización sustentable de los recursos naturales básicos en la producción, de manera benévola, de productos agropecuarios alimentarios y no alimentarios inocuos y saludables (8). El Continente Americano en el ámbito mundial, es el primer productor de carne bovina, tercero de carne porcina y primero en producción láctea, citando los más destacados. Así comparando con otros continentes, ostenta la menor concentración de superficie por habitante y población animal (9). Por lo anterior, las BPG contribuyen a enfrentar con éxito las nuevas demandas de consumo y comercialización de productos pecuarios, permitiendo dar seguimiento a la calidad e inocuidad del producto en la cadena alimentaria y vigilar que no contengan residuos que afecten el medio 
ambiente, arriesguen la salud de la población consumidora, productora, y se cuide del bienestar de los animales (10).

Concretamente las BPG cubren los siguientes aspectos $(8,10)$ :

1. Control de entrada de animales nuevos.

2. Medidas de higiene sobre el alimento y el agua para los animales.

3. Control de contacto con otras explotaciones dentro de la misma granja

4. Control de vectores y animales domésticos.

5. Control de fómites.

6. Manejo del estiércol.

7. Manejo del Personal.

Como objetivo general se tuvo estimar la prevalencia de Cryptosporidium spp. y Giardia spp. en terneros de 0 a 2 meses de ganado lechero de la Sabana de Bogotá y sus factores de riesgo asociados mediante un estudio de corte transversal. Los objetivos específicos fueron estimar los factores de riesgo de la producción lechera en cuanto a buenas prácticas de ganadería que puedan incurrir en la infección de personas y animales.

\section{MATERIALES Y METODOS}

Se realizó un estudio epidemiológico de corte transversal para estimar la prevalencia de Giardia spp. y Cryptosporidium spp. en 308 terneros de razas productoras de leche en 33 fincas del noroccidente de la Sabana de Bogotá durante el periodo comprendido entre Marzo y Octubre del año 2009. La muestra utilizada fue materia fecal obtenida directamente de la ampolla rectal. Debido a la naturaleza zoonótica de estos protozoarios y de su importancia en Salud Pública, se realizó el cálculo de Odds Ratio (OR) para cada parásito, con el fin de determinar posibles factores de riesgo asociados a la infección, al manejo sanitario del hato y a la producción de leche.

Se muestrearon animales de la especie Bos taurus de razas lecheras como Holstein, Normando, Jersey, Ayrshire y sus cruces. Los animales seleccionados tuvieron entre 0 y 2 meses de edad, debido a que es el periodo más susceptible para la infección de Giardia spp. y Cryptosporidium spp (11-13). De acuerdo a los datos arrojados por la Encuesta Nacional Agropecuaria del 2007, en Cundinamarca la población estimada de bovinos 
destinados a la producción de leche entre los 0 y 12 meses de edad fue de 26124 (7). Ya sin datos discriminados para la Sabana de Bogotá ni para el rango de edad con que se diseñó el estudio, se utilizó el dato de 26.124 como la población universo de terneros. La prevalencia esperada calculada para el estudio fue de $40 \%$ debido a que es el valor reportado con mayor frecuencia, tanto como para Giardia spp. (2,5,14-17) como para Cryptosporidium spp. $(2,11,18,19,20)$. Se manejó un nivel de confianza del $5 \%$ y un error relativo del $15 \%$.

Se obtuvieron aproximadamente $40 \mathrm{~g}$ de materia fecal en un guante plástico para palpación rectal. En el cual se almacenó la muestra, se selló con cinta adhesiva y se marcó con el número de identificación del ternero, sexo y el nombre de la finca. Las muestras fueron refrigeradas y transportadas en un recipiente a $4^{\circ} \mathrm{C}$ en donde no las afectó la luz solar. En el Laboratorio de Parasitología Veterinaria de la Universidad Nacional de Colombia (LPV-UN) se almacenaron las muestras en bolsas plásticas, debidamente marcadas con el nombre del estudio y la fecha del muestreo en un refrigerador $4^{\circ} \mathrm{C}$. Las muestras fueron procesadas en el LPV-UN. Se utilizó la técnica de Ritchie (Formol-Éter) para establecer la presencia de quistes de Giardia spp. (14, 15, 21) y la coloración de Ziehl-Neelsen Modificada para establecer la presencia de ooquistes de Cryptosporidium spp (11, 18, 22). Para observar los quistes de Giardia spp. y los ooquistes de Cryptosporidium spp, se utilizó un microscopio óptico (Olympus ${ }^{\circledR}$ CH30 con objetivos de $4 \mathrm{X}, 10 \mathrm{X}, 40 \mathrm{X}$ y $100 \mathrm{X}$ y oculares de $10 \mathrm{X}$ Nikon ${ }^{\circledR}$ E200 con objetivos de 4X, 10X, 40X y 100X y oculares de 10X).

Se diseñó un cuestionario, para evaluar las BPG de cada finca. La información obtenida se cruzó con los resultados obtenidos en los coprológicos para poder determinar los factores de riesgo. Una vez obtenidos los resultados de las muestras de materia fecal, se creó una base de datos utilizando el programa Excel ${ }^{\circledR} 8.0$ en donde se tabuló la información obtenida en la visita a cada finca mediante el cuestionario enfrentada a los resultados arrojados por las técnicas coprológicas de cada uno de los animales muestreados. Ya finalizada la base de datos se procedió a realizar los cálculos de OR cruzando la información del manejo de la finca con los resultados de los coprológicos para poder determinar posibles factores de riesgo. Para el calculo de los OR se utilizó el programa estadístico Epi Info $^{\circledR}$ 3.5.1. 


\section{RESULTADOS}

Se incluyeron en el muestreo 308 terneros entre 0 y 2 meses de edad de 33 fincas dedicadas a la ganadería lechera en la zona noroccidental de la Sabana de Bogotá. Por dificultad en la obtención de los registros catastrales de los municipios, no se llevó a cabo un muestreo aleatorio. La selección de las fincas se realizó por la técnica de muestreo por conveniencia. El método de selección aleatoria muestra aplicado a los terneros fue el muestreo por conglomerados, de manera que se seleccionaron los terneros de cada finca pertenecientes al grupo etario (0-2 meses).

De los 308 terneros muestreados 237 (76,9 \%) eran de la raza Holstein, 32 (10,4\%) eran de la raza Normando, 11 (3,6 \%) de la raza Ayrshire, 10 (3,2 \%) de la raza Pardo Suizo, 7 (2,3 \%) de la raza Jersey y el 3,4 \% restante comprendió cruces de Holstein con otras. Se obtuvo un mayor número de terneros muestreados en el rango de 11-20 días, con un total de 72 terneros (23,4 \%), 59 (19,2\%) de los cuales pertenecían al rango de 21-30 días, 53 (17,2 \%) al rango de 31-40 días, 47 (15,3\%) al rango de 0-10 días, 42 (13,6 \%) al rango de 51-60 días 35 (11,4\%) al rango de 41-50 días. Un total de 286 (92,9 \%) de los animales seleccionados fueron hembras y 21 (7, $1 \%)$ correspondieron a machos.

La prevalencia de punto de Giardia spp. para los 308 terneros entre 0 y 2 meses de edad, de 33 fincas de la zona noroccidental de la Sabana de Bogotá, fue de 37,3 \%; esto quiere decir que en 115 animales de 308 muestreados se observaron quistes de Giardia por el método coprológico de Ritchie (Tabla 1).

Tabla 1. Resultados técnica de Ritchie para Giardia spp. (n=308)

\begin{tabular}{lccccc}
\hline \multirow{2}{*}{ Item } & \multirow{2}{*}{ Negativos } & \multicolumn{4}{c}{ Positivos } \\
\cline { 3 - 6 } & & Total & \multicolumn{3}{c}{ Carga parasitaria } \\
& & positivos & Alta & Media & Baja \\
\hline Número de animales & 193 & 115 & 26 & 39 & 50 \\
Porcentaje & 62,7 & 37,3 & 22,6 & 33,9 & 43,5 \\
\hline
\end{tabular}

La prevalencia de punto de Cryptosporidium spp. para 308 terneros de 0 a 2 meses de edad, en 33 fincas de la zona noroccidental de la Sabana de Bogotá fue de 4,9\%; en 15 animales de los muestreados se observaron ooquistes de Cryptosporidium spp., utilizando el método de Ziehl-Neelsen modificado (Tabla 2). 
Tabla 2. Resultados coloración Ziehl-Neelsen para Cryptosporidium spp ( $\mathrm{n=308)}$

\begin{tabular}{cccccc}
\hline & \multirow{2}{*}{ Negativos } & \multicolumn{4}{c}{ Positivos } \\
\cline { 3 - 6 } & & Total & \multicolumn{3}{c}{ Carga parasitaria } \\
& positivos & Alta & Media & Baja \\
\hline Número de animales & 293 & 15 & 0 & 7 & 8 \\
Porcentaje & 95 & 4,9 & 0 & 46,7 & 53,3 \\
\hline
\end{tabular}

Como se observa en la Tabla 3 los valores de OR, para presencia de gatos representa un riesgo tres veces mayor de infección por Giardia spp. y la presencia de asnos incrementa nueve veces el riesgo de infección por Cryptosporidium spp. con relación a las fincas que no reportan la presencia de los mismos. En la misma tabla se observa que el valor de OR en ovinos/ caprinos para Giardia spp. es de 0,26 y en asnos es de 0,11 indicando que la presencia de estas dos especies de protozoarios podría constituir un factor de protección para la infección por este parásito, aunque estos valores son estadísticamente significativos, contradicen los reportes que demuestran que Giardia spp., infecta a todos los mamíferos $(1,3,6,19)$.

Tabla 3. Presencia de otros animales en las fincas y valores de OR para los dos protozoarios

\begin{tabular}{lcccccc}
\hline \multicolumn{1}{c}{ ESPECIE } & $\begin{array}{c}\text { Número de } \\
\text { fincas }\end{array}$ & $(\%)$ & O.R & IC. $95 \%$ & O.R & IC $95 \%$ \\
\hline Felinos & $13 / 33$ & 39,3 & 3,59 & $2,21-5,82$ & 1,94 & $0,69-5,51$ \\
Asnales & $3 / 33$ & 9,1 & 0,11 & $0,01-0,86$ & 9,2 & $2,54-33,85$ \\
Ovinos/Caprinos & $8 / 33$ & 24,2 & 0,26 & $0,13-0,51$ & 0,82 & $0,23-0,37$ \\
\hline
\end{tabular}

El 24,3 \% de las fincas muestreadas reportó la compra de animales (Tabla 4).

De acuerdo con el valor de OR para Cryptosporidium spp., en la Tabla 4 , comprar animales representa un riesgo tres veces mayor para la infección con este parásito.

Tabla 4. Compra de animales y valores de OR relacionados para los dos protozoarios

\begin{tabular}{cccrccc}
\hline \multirow{2}{*}{ Compra de animales } & $\begin{array}{c}\text { Número de } \\
\text { Fincas }\end{array}$ & \multirow{2}{*}{$(\%)$} & \multicolumn{2}{c}{ Giardia } & \multicolumn{2}{c}{ Cryptosporidium } \\
& OR. & IC. $95 \%$ & OR. & IC. $95 \%$ \\
\hline Si compran & $8 / 33$ & 24,3 & 1,36 & $0,82-2,25$ & 2,97 & $1,04-8,47$ \\
\hline
\end{tabular}

De acuerdo con la Tabla 5, los valores de OR para Giardia spp., muestran que en las fincas que obtienen el agua de vallados hay un riesgo tres veces 
mayor de infección y en las fincas que obtienen el agua de otras fuentes (quebrada, laguna, nacimientos, lagos), existe un riesgo dos veces mayor de infección con respecto a las que no lo hacen. De otro lado, el valor de OR $(0,29)$, para Giardia spp., en las fincas que obtienen el agua de pozo profundo, indica que puede ser un factor de protección contra la infección por este protozoario, debido a que está menos expuesta al contacto con las heces.

Sin embargo el valor de OR para Cryptosporidium spp., en las fincas que obtienen el agua de pozo profundo indica la existencia de un riesgo seis veces mayor con respecto a las fincas que no lo hacen, no coincidiendo con lo observado en Giardia spp., probablemente esto se deba a que la contaminación del agua con excretas, no se genere directamente en la fuente.

Tabla 5. Origen del agua suministrada y valores de OR relacionados para los dos protozoarios

\begin{tabular}{ccccccc}
\hline $\begin{array}{c}\text { Origen del } \\
\text { agua }\end{array}$ & $\begin{array}{c}\text { Numero de } \\
\text { fincas }\end{array}$ & $(\%)$ & OR & IC 95\% & OR & IC 95\% \\
\hline Vallado & $13 / 33$ & 39,4 & 2,77 & $1,62-4,72$ & 1,13 & $0,35-3,66$ \\
Pozo & $16 / 33$ & 48,5 & 0,29 & $0,17-0,47$ & 5,82 & $1,29-26,27$ \\
Acueducto & $8 / 33$ & 24,2 & 0,62 & $0,36-1,08$ & 1,5 & $0,5-4,53$ \\
Otros & $6 / 33$ & 18,2 & 1,7 & $1,06-2,83$ & 1,47 & $0,51-4,26$ \\
$\begin{array}{c}\text { Realizan } \\
\text { tratamiento }\end{array}$ & $10 / 33$ & 30,3 & 0,17 & $0,09-0,34$ & 0,26 & $0,16-0,68$ \\
\hline
\end{tabular}

A partir de la Tabla 5 el 69,7 \% de las fincas muestreadas no realiza ningún tratamiento al agua, sin tener en cuenta las BPG recomendadas por Giménez (10) y Vargas-Terán (8). Estos autores sostienen que si no es posible obtener el agua de las redes de acueducto es conveniente hacer algún tipo de tratamiento. De acuerdo con los valores de OR obtenidos en el presente estudio para Giardia spp. $(0,17)$ y Cryptosporidium spp. $(0,26)$, el 30,3 \% de las fincas muestreadas que realizan algún tipo de tratamiento al agua, obtienen un factor de protección contra la infección por estos dos parásitos.

Según lo observado en la Tabla 6, el 21,2 \% de las fincas muestreadas no realizan ningún tipo de tratamiento a las excretas animales aumentando así, la probabilidad de infección a tres veces para Giardia spp., y a dos veces para Cryptosporidium spp. con respecto a las fincas que realizan algún tipo de tratamiento. El 27,3 \% de las fincas muestreadas esparcen las excretas 
en las praderas con fin de usarlo como abono, incrementando dos veces, el riesgo de infección para Giardia spp.

Tabla 6. Manejo de Excretas $(n=33)$

\begin{tabular}{|c|c|c|c|c|c|c|}
\hline \multirow{2}{*}{$\begin{array}{c}\text { Manejo de las } \\
\text { excretas }\end{array}$} & \multirow{2}{*}{$\begin{array}{l}\text { Número } \\
\text { de fincas }\end{array}$} & \multirow{2}{*}{ (\%) } & \multicolumn{2}{|c|}{ Giardia } & \multicolumn{2}{|c|}{ Cryptosporidium } \\
\hline & & & OR & IC 95\% & OR & IC 95\% \\
\hline Ninguno & 7 & 21,2 & 2,79 & $1,57-4,95$ & 1,5 & $0,46-0,88$ \\
\hline Abono & 9 & 27,3 & 1,14 & $1,06-2,03$ & 1,14 & $0,66-5,31$ \\
\hline Compost & 4 & 12,1 & 0,93 & $0,53-1,63$ & 0,86 & $0,23-3,12$ \\
\hline Pozo estercolero & 11 & 33,3 & 0,61 & $0,37-0,82$ & 1,87 & $0,36-0,82$ \\
\hline Otros & 2 & 6,1 & & & & \\
\hline
\end{tabular}

En la utilización de pozo estercolero por el 33,3 \% de las fincas muestreadas, puede ser factor de protección para la infección por Giardia spp. Sin embargo, para el caso de Cryptosporidium spp. el valor de OR indica que la utilización del pozo estercolero incrementa dos veces el riesgo de infección.

Se observa en la Tabla 7 el 84,8 \% de las fincas muestreadas cuentan con algún tipo de equipo de ordeño y de acuerdo con el valor de OR para Giardia spp. $(0,16)$ y para Cryptosporidium spp. $(0,45)$, este equipo puede ser un factor de protección.

Tabla 7. Presencia de equipos y sus valores de OR para Giardia spp. y Cryptosporidium spp.

\begin{tabular}{lcccccc}
\hline \multirow{2}{*}{ Equipos } & $\begin{array}{c}\text { Número de } \\
\text { fincas }\end{array}$ & \multirow{2}{*}{$(\%)$} & \multicolumn{2}{c}{ Giardia } & \multicolumn{2}{c}{ Cryptosporidium } \\
& OR & IC 95\% & OR & IC 95\% \\
\hline Tanque de enfriamiento & $11 / 33$ & 33 & 0,28 & $0,15-0,51$ & 0,3 & $0,1-0,91$ \\
Equipo de ordeño & $28 / 33$ & 84,8 & 0,16 & $0,05-0,46$ & 0,45 & $0,09-2,15$ \\
\hline
\end{tabular}

Los datos de la Tabla 8, indican que el $97 \%$ de las fincas muestreadas, habitan trabajadores y los valores de OR muestran que vivir en la finca incrementa siete veces el riesgo de infección por Giardia spp y representa un riesgo de infección por Cryptosporidium spp. de cuatro veces para los trabajadores, con respecto a las fincas donde esto no ocurre.

Los datos de la Tabla 9, indican que el 42,4 \% de las fincas muestreadas cuentan con un tipo de asistencia técnica mixta, la realiza personal particular y personas que representan a algún tipo de casa comercial. Del valor de OR para Giardia spp. $(0,5)$, se infiere que este tipo de asistencia técnica 
protege contra la infección por parte de este patógeno con respecto a las fincas que no cuentan con este servicio. También se observa en la Tabla 9, referente a la frecuencia de la asistencia técnica un 12,2 \% de las fincas muestreadas cuentan una frecuencia eventual; el OR para Giardia spp. indica que esta frecuencia en la asistencia, representa 14 veces más riesgo para la infección por este parásito, con respecto a las fincas que no cuentan con esta frecuencia de asistencia. La Tabla 9, muestra que el 39,4 \% de las fincas seleccionadas cuentan con asistencia técnica permanente y el valor de OR para Giardia spp. $(0,53)$ y Cryptosporidium spp. $(0,57)$, señala que esta frecuencia en el tipo de asistencia protege contra la infección por estos parásitos. Referente al tipo de persona que presta la asistencia técnica (Tabla 9), el 93,9 \% de las fincas muestreadas cuentan con un profesional en la asistencia y los valores de OR para Giardia spp. $(0,74)$ y Cryptosporidium spp. $(0,08)$, indican que este hecho protege en contra de la infección.

Tabla 8. Trabajadores que viven en la finca y valores de OR para Giardia spp. y Cryptosporidium spp. $(\mathrm{n}=33)$

\begin{tabular}{lcccccc}
\hline $\begin{array}{l}\text { Trabajadores que viven } \\
\text { en las fincas }\end{array}$ & $\begin{array}{c}\text { Número de } \\
\text { fincas }\end{array}$ & (\%) & OR & IC 95\% & OR & IC 95\% \\
\hline Si viven en la finca & 32 & 97 & 6,6 & $1,97-2,33$ & 4,22 & $2,03-3,87$ \\
No viven en la finca & 1 & 3 & 0,39 & $0,06-2,38$ & 0,28 & $0,08-0,76$ \\
\hline
\end{tabular}

Tabla 9. Asistencia técnica y valores de OR para Giardia y Cryptosporidium

\begin{tabular}{lcccccc}
\hline \multirow{2}{*}{ Tipo } & $\begin{array}{c}\text { Número de } \\
\text { fincas } \\
(\mathrm{n}=33)\end{array}$ & $(\%)$ & & \multicolumn{2}{c}{ Giardia } & \multicolumn{2}{c}{ Cryptosporidium } \\
\cline { 5 - 7 } & 15 & 45,4 & 1,57 & $0,97-2,53$ & 0,41 & $0,11-0,59$ \\
\hline Particular & 4 & 12,2 & 1,46 & $0,81-2,61$ & 2,25 & $0,74-6,86$ \\
Comercial & 14 & 42,4 & 0,5 & $0,31-0,81$ & 1,1 & $0,39-3,12$ \\
Mixta & & & & & & \\
Frecuencia & 4 & 12,2 & 14,25 & $3,19-63,5$ & & \\
Eventual & 16 & 48,4 & 1,14 & $0,71-1,83$ & 2,21 & $0,76-6,37$ \\
Periódica & 13 & 39,4 & 0,53 & $0,35-0,85$ & 0,57 & $0,19-0,65$ \\
Permanente & & & & & & \\
Quien asiste & 31 & 93,9 & 0,74 & $0,19-0,82$ & 0,08 & $0,02-0,37$ \\
Profesional & 6 & 18,2 & 0,76 & $0,44-1,31$ & 1,1 & $0,32-3,41$ \\
\hline Tecnólogo & & & & & &
\end{tabular}

\section{DISCUSION}

La prevalencia de Giardia spp. encontrada en este estudio concuerda con los datos reportados en otras partes $(2,12,14-17,23)$. Anotando que los 
animales positivos (33 \%, $\mathrm{n}=115)$ presentaron diarrea en el momento de la toma de la muestra. Se observó que un 22,6 \% de los animales muestreados tuvieron una carga alta (Tabla 1), de quistes de Giardia spp. de los cuales la totalidad presentó diarrea, lo que pudo ser una manifestación clínica de la infección. El 43,5 \% de los animales positivos tuvieron una carga baja de quistes, y se observaron asintomáticos. Este grupo de animales podría constituir en una población reservorio para los demás animales.

La prevalencia de Cryptosporidium spp. obtenida en el presente estudio (Tabla 2) coincide con el rango entre 3 a 40 \% (2). Sin embargo, la prevalencia no se encuentra dentro del valor reportado por otros investigadores $(11,17,18,20)$, que se utilizó como valor esperado en el diseño de este estudio. Dicha diferencia posiblemente se origina por factores como el comportamiento epidemiológico del parásito. Esto quiere decir que al ser el presente estudio de tipo transversal, probablemente encontró al parásito en una etapa inicial de su proceso de reemergencia. También, es posible que la baja prevalencia obtenida para este protozoario, se deba a que el mayor porcentaje de terneros muestreados se encontró en el grupo etario de 11-20 días y el grupo etario más susceptible a la infección por Cryptosporidium spp., es de 0-7 días según lo reportado $(1,11,19,24)$.

Es evidente que el cumplimiento de las BPG (8-10) disminuye los factores de riesgo en la transmisión de estos dos parásitos tanto en los demás animales de producción como en el personal que trabaja y vive en las fincas. Se debe ser consciente que más del $85 \%$ de los alimentos producidos en las granjas, se comercializa, se distribuye y se expende para el consumo de la población según sus necesidades; por lo tanto es imperativa la inocuidad de estos productos y la buena condición sanitaria de los animales que los producen (25).

De acuerdo a los resultados arrojados por éste estudio; se encontraron los siguientes factores de protección:

- Asistencia técnica permanente bajo criterio de un profesional.

- Tratamiento al agua de suministro.

- Compostaje de excretas.

- Equipo de ordeño y tanque de enfriamiento. 
Se recomienda el planteamiento y la realización de más estudios que amplíen el conocimiento en la problemática planteada en esta investigación, determinar más factores de riesgo o profundizar en los mismos y estudiar el comportamiento a lo largo de un periodo de tiempo

\section{REFERENCIAS}

1. Acha P, Szyfres B. Zoonosis y enfermedades trasmisibles comunes al hombre y a los animales. Pub. Científica $N^{\circ} 503,5^{a}$ Ed. Organización Panamericana de la Salud OPS. 1996.

2. World Health Organization. Risk assessment of Cryptosporidium in drinking water, Sidney WHO/HSE/WSH/09.04; 2009: 6-78.

3. Alcazar D. Giardia y Giardiosis. Control y Calidad SEIMC; 2008.

4. Spano F, Crisanti A. Cryptosporidium parvum: the many secrets of a small genome. Int $\mathrm{J}$. Parasitol 2004;:553-365.

5. Luján HD, Mowatt MR, WU Jl. Purification of a variant-specific surface protein of Giardia lamblia and characterization of its metal-binding properties. J Bio Chem. 2009; 270: 13807-13.

6. Kulda J, Nohynkova E. Giardia in humans and animals. En: Kreier JP (ed.) Parasitic protozoa $2^{\mathrm{a}}$ ed. San Diego: Academic Press, 2006; 10:225-242.

7. Ministerio de Agricultura y Desarrollo Rural. Encuesta Nacional Agropecuaria. Colombia; 2007: 81-82.

8. Vargas-Teran M. Buenas prácticas ganaderas. Food and Agriculture Organitation (FAO). Chile; 2009. pp. 1-5.

9. Arriaga A. Seguridad sanitaria en granjas de rumiantes. Departamento de Agricultura, Ganadería y Alimentación, Sección de Sanidad Animal. España; 2003. pp. 55-59.

10. Gimenez M. Manual de buenas prácticas ganaderas. Sitio Argentino de Producción Animal. Argentina; 2006. pp.1-10.

11. Dupont HL, Chappell CL, Sterling CR, Okhuysen PC, Rose JB, Jakubowski W. The infectivity of Cryptosporidium parvum in healthy volunteers. N Engl J Med. 1995; 332:855-859.

12. Araujo W, Chávez A, Casas E, Falcón N. Prevalencia de Giardia spp. en Canis familiaris de los Distritos de la Provincia Constitucional del Callao. Revista de Investigación Veterinaria Perú. 2009; 15 (2): 145-150.

13. Rodriguez JC, Royo G. Cryptosporidium y criptosporidiosis. Control y Calidad SEIMC; 2008.

14. Navarro C, Peris B, Garijo MY, Gómez MT. Estudio epidemilógico de Giardia spp y Eimeria spp en el ganado bovino de la Comunidad Valenciana. Factores de riesgo. SEOC Patología y Sanidad Animal. 2008: 320-323.

15. Appelbee AJ, Frederic LM, Heitman TL, Olson ME. Prevalence and genotyping of Giardia duodenalis from beef calves in Alberta, Canada. Vet. Parasitol, 2009; 112: 289-294.

16. Zárate D, Chávez A, Casas E, Falcón N. Prevalencia de Giardia sp. en canes de los distritos de Cono Sur de Lima Metropolitana. Rev Inv Vet Perú. 2008; 14 (2): 134 139.

17. Solarte $Y$, Peña M, Madera C. Transmisión de protozoarios patógenos a través del agua para consumo humano. Colombia Médica. 2006; 37 (1): 74-82.

18. Castro-Hermida JA, González-Losada A, Ares-Mazás E. Prevalence of and risk factors involved in spread of neonatal bovine cryptosporidiosis in Galicia (NW Spain). Vet. Parasitology. 2008; 106: 1-10. 
19. Morgan UM, Constantine CC, Forbes DA, Thompson RC. Differentiation between human and animal isolates of Cryptosporidium parvum using rDNA sequencing and direct PCR analysis. J Parasitol. 1997; 83:825-830.

20. Valera Z, Quintero W, VIllarroel R. Hernández E. Cryptosporidium sp. en una finca del municipio Rosario del Perijá, Estado Zulia, Venezuela. Revista Cientifica FCV-LUZ. 2009; XXI (3): 213-218.

21. Trout JM, Santin M, Greiner E, Fayer R. Prevalence of Giardia duodenalis genotypes in pre-weaned dairy calves. Vet. Parasitol. 2009; 124: 179-186.

22. Farthing MG. Clínica y Tratamiento de las Infecciones Intestinales por Protozoos. Nature Clinical Practice Gastroenterology and Hepatology. 2006; 3(8):436-445.

23. Ruest N, Faubert GM, Couture Y. Prevalence and geographical distribution of Giardia spp. and Cryptosporidium spp. in dairy farms in Quebec. Can. Vet. J. 2007; 39: 697-700.

24. Becher K.A, Robertson ID, Fraser DM, Palmer DG, Thompson RCA. Molecular epidemiology of Giardia and Cryptosporidium infections in dairy calves originating from three sources in Western Australia. Veterinary Parasitology.2004; 123: 1-9.

25. O'donoghue PJ. Cryptosporidium and cryptosporidiasis in man and animals. Int $\mathrm{J}$ Parasitol. 1995; 25:139-195. 